\title{
Electron population of impurity atoms in crystal in external quantizing magnetic field
}

\author{
B.A.Lukiyanets \\ State University "Lvivs'ka Politekhnika", \\ 12 Bandera Str., 79646 Lviv, Ukraine
}

Received July 3, 2002

\begin{abstract}
The model which is discussed permits to describe the charge state of the impurity both at the intercalation of a layer crystal and at the adsorption on a crystal surface. In the framework of the model, the influence of the external quantizing magnetic field on the magnitude of charge population of the impurity is analyzed. The obtained results indicate that the external magnetic field is an important factor that influences the effects of adsorption or intercalation.
\end{abstract}

Key words: impurity atom, intercalation, adsorption, quantizing magnetic field

PACS: $61.72 . B b, 68.35 . D$

\section{Introduction}

The improvement of an impurity atom into a crystal matrix is inevitably connected with the charge transfer from the impurity into a crystal matrix or vise-versa. Such a transfer can sometimes considerably change physical characteristics of the crystal. In particular, it can radically modify the topology of the Fermi surfaces of the metal compounds and, in some cases, to the extent of including a metal-semiconductor phase transition [1]. The crystals with adsorbed atoms on their surfaces as well as the intercalated layer crystals belong to those particularly sensitive to such a transfer. In the latter ones, electronic transfer from the intercalated atoms into the crystal matrix takes place. The alkali atoms are the most effective electron donors. They may supply up to one electron to the crystal for each formula unit [2]. As a result, a weak interlayer interaction (which determines a quasi-dimensional character of the layer crystal) is placed by strong - Coulomb or covalent - interaction and thus the intercalated layer crystal becomes more "three-dimensional". Drawing a parallel between the electron transfer from an impurity atom in intercalation of a layer crystal and the electron transfer in adsorption has a more deep justification 
than it may seem at first glance. Really, the distribution of the intercalated atoms has a two-dimensional character - they create an additional atomic plane in the van der Waals gap of the layer crystal. Such atoms resemble the atoms adsorbed on the crystal surface. From this point of view, a layer crystal may be identified with a crystal having a sufficiently developed surface in $\sim N_{z}$ times more than in the volume crystal ( $N_{z}$ is a number of layers along the C-axis of a crystal lattice). Such an analogy becomes still more obvious if the interlayer electron mixing $\beta$ tends to zero. In this case the layer crystal breaks up into a set of independent layers and intercalation can be considered as an adsorption. The only difference is that the adsorbed atoms settle on a semiinfinite crystal but not on the layers - plates with the width equal to a lattice parameter of the layer crystal. In a general case, $\beta \neq 0$,the intercalation appears to be a volume effect and the electron spectrum herein can be obtained from Schrodinger equation with Born-Karman's conditions. But adsorption is the effect connected with a crystal surface and therefore in that case the eigenvalue problem is more complicated. In particular, at certain conditions the surface states are observed. Aside from this difference, the proposed analogy between an intercalated crystal and an adsorbate-adsorbent system is quite obvious: atomic planes of a layer crystal limited by van der Waals gap resemble the surface of an adsorbate, and intercalated atoms resemble the adatoms. Therefore, the same model can be used in the both cases to solve the problem of the electron population of the impurity atoms. First microscopic analysis of the behaviour of the adsorbed atoms on a crystal surface were presented in several papers [3-6]. There were the same models in the papers. According to the model, the one-electron wave functions of the adsorbate-adsorbent system are presented as a linear combination of the free impurity atoms and free crystal functions. Hence, the interaction between the impurity and the crystal matrix lies in the fact that each original discrete atomic level acquires a certain width. The Hamiltonian of the model is of the first type used by Anderson [7] in the theory of dilute alloys. The model analysis enables us to get a deeper insight into the adsorption, in particular, to investigate an adsorption energy, an energy shift and other experimentally detected characteristics. The purpose of this paper is to study the effect of the external quantizing magnetic field on the electronic population (occupancy) of the impurity in the system "two-impurity (adsorbed or intercalated atoms)+crystal matrix" within the framework of the modified Anderson's Hamiltonian:

$$
\widehat{H}=\sum_{k} \varepsilon_{k} c_{k}^{+} c_{k}+E_{0}\left(a_{0}^{+} a_{0}+a_{1}^{+} a_{1}\right)+\sum_{\alpha k} V(\alpha k)\left(c_{k}^{+} a_{\alpha}+\text { h.c. }\right)+U n_{0} n_{1} .
$$

Here, the first term describes a free crystal in the external magnetic field $\vec{H}$, directed along $O Z$-axis (let $O Z$ be a perpendicular to a crystal surface or to a layer). Then,

$$
\varepsilon_{k}=L_{0}+\hbar \omega_{c}\left(n+\frac{1}{2}\right)+\frac{\hbar^{2} k_{z}^{2}}{2 m_{z}^{*}}
$$

where $L_{0}$ is the bottom of the band, $k=\left(n, k_{z}\right)$ ( $n$ is the number of Landau levels; lattice parameter $d_{z}$ is taken equal unit), $\omega_{c}=e H / m^{*} c\left(m^{*}\right.$ is an effective mass 
of the electron in the plane of the layers). The second term in (1) describes the free impurity atoms; $a_{\alpha}^{+}, a_{\alpha}(\alpha=0,1)$ are the Fermion creation and annihilation operators, $n_{\alpha}=a_{\alpha}^{+} a_{\alpha}$ is the corresponding number operator $\left(c_{k}^{+}, c_{k}\right.$ and $n_{k}$ have the same meaning for the crystal). The third term is simply an electron overlapping between the atoms and the crystal matrix, and the fourth term is the inter-atomic Coulomb repulsion between the impurities.

Let us note the differences between Hamiltonian (1) and the Hamiltonian of the cited papers. Firstly, since Hamiltonian (1) will be used for a description of the behaviour of the impurity atom in a crystal matrix in the quantizing magnetic field, the spin indexes are omitted. Secondly, as in the cited papers, we neglected all but Coulomb repulsion of the impurities. However, we consider the interatomic interaction in contrast to the mentioned papers, in which an intraatomic Coulomb repulsion of electrons with opposite spins is considered. This is partly justified in the adsorption by the fact that relatively simple adsorbates like $\mathrm{H}, \mathrm{O}$ or the halogens have a larger value of the Coulomb interaction than the crystal matrix due to the screening in the crystal and by the spatial distribution of the adsorbed (intercalated as well) atoms. A similar problem in the system without magnetic field within the framework of Hartree-Fock approximation was considered in [8]. In this paper we from the very beginning analyze the effect of the magnetic field in the mentioned approximation and further we investigate the problem within the framework of the alternative approximation. We restrict ourselves to the case of a low temperature at which the effect of the magnetic field is the most clear.

\section{Electronic population of an impurity. Hartree-Fock approximation}

Let us introduce the double-time retarded Green's function $\left\langle\left\langle a_{\alpha} \mid a_{\alpha^{\prime}}^{+}\right\rangle\right\rangle\left(\alpha, \alpha^{\prime}=\right.$ $0,1)$, which particularly contains the information about the average number of electron population of the impurity atoms $\left\langle n_{0}\right\rangle,\left\langle n_{1}\right\rangle$. Using the equation-of-motion method to the Green's function in the Hartree-Fock approximation, i.e.

$$
n_{0} n_{1}=\left(n_{0}\left\langle n_{1}\right\rangle+n_{1}\left\langle n_{0}\right\rangle\right) / 2
$$

we obtain the following equations:

$$
\begin{aligned}
\left(\omega-E_{0}-\frac{U}{2}\left\langle n_{1}\right\rangle-W_{00}\right)\left\langle\left\langle a_{0} \mid a_{0}^{+}\right\rangle\right\rangle-W_{01}\left\langle\left\langle a_{1} \mid a_{0}^{+}\right\rangle\right\rangle & =1, \\
-W_{10}\left\langle\left\langle a_{0} \mid a_{0}^{+}\right\rangle\right\rangle+\left(\omega-E_{0}-\frac{U}{2}\left\langle n_{0}\right\rangle-W_{11}\right)\left\langle\left\langle a_{1} \mid a_{0}^{+}\right\rangle\right\rangle & =0
\end{aligned}
$$

where

$$
W_{\alpha \alpha^{\prime}}=\sum_{k} \frac{V^{*}(\alpha, k) V\left(\alpha^{\prime}, k\right)}{\omega-\varepsilon_{k}}
$$

is a characteristic, which contains information about both crystal matrix and its electron sharing with the impurity. In the widely used assumption $W_{\alpha \beta}=$ const, the 
information about the crystal matrix in the problem under consideration is contained in the chemical potential of the crystal-impurity system only. The equation for $\mu$ in the problem described by the Hamiltonian (1) can be written as follows:

$$
n=\sum_{k} f\left(\tilde{\varepsilon}_{k}\right)+f\left(E_{1}\right)+f\left(E_{2}\right),
$$

where $n$ is a concentration of electrons of the system, $\tilde{\varepsilon}_{k}$ is an electron spectrum in the crystal matrix renormalized by the impurities, $f(x)$ is Fermi-Dirac distribution function, and $E_{1}, E_{2}$ are the electron states of the impurities obtained as the solution of the equality to the zero of the coefficient determinant of the system of linear equations (3), namely:

$$
\left(\omega-E_{0}-\frac{U}{2}\left\langle n_{1}\right\rangle-W_{00}\right)\left(\omega-E_{0}-\frac{U}{2}\left\langle n_{0}\right\rangle-W_{11}\right)-\left|W_{01}\right|^{2}=0,
$$

i.e.

$$
E_{s}=\left(E_{0}+\frac{1}{2}\left(W_{00}+W_{11}\right)+\frac{U}{4}\left(\left\langle n_{0}\right\rangle+\left\langle n_{1}\right\rangle\right)\right)+(-1)^{s+1} K, \quad s=1,2
$$

with

$$
K=\frac{1}{2} \sqrt{\left[\left(W_{00}-W_{11}\right)^{2}+\frac{U}{2}\left(\left\langle n_{0}\right\rangle-\left\langle n_{1}\right\rangle\right)\right]^{2}+4\left|W_{01}\right|^{2}}
$$

In the two-impurity problem the contribution of two last terms in (4) cannot exceed 1 and their value is $O(1 / N)$ ( $N$ is a number of sites in the crystal). Thus, in the zeroth approximation, the value of the chemical potential is defined by the crystal matrix. Furthermore, in this case one can ignore the renormalization of the crystal matrix spectrum by two impurities.

From the system of equations (3) it follows:

$$
\left\langle\left\langle a_{0} \mid a_{0}^{+}\right\rangle\right\rangle=\frac{\omega-E_{0}-\frac{U}{2}\left\langle n_{0}\right\rangle-W_{11}}{\left(\omega-E_{1}\right)\left(\omega-E_{2}\right)},
$$

and

$$
\left\langle\left\langle a_{1} \mid a_{0}^{+}\right\rangle\right\rangle=\frac{W_{10}}{\left(\omega-E_{1}\right)\left(\omega-E_{2}\right)} .
$$

The form of the Green's functions $\left\langle\left\langle a_{1} \mid a_{1}^{+}\right\rangle\right\rangle$and $\left\langle\left\langle a_{0} \mid a_{1}^{+}\right\rangle\right\rangle$coincides with Green's functions (8), (9) respectively, after the formal change of the index 0 to 1 and viceversa.

Let us calculate the average number of electron population of the impurities. It is known that

$$
\langle n\rangle=\int \rho(\omega) f(\omega) \mathrm{d} \omega
$$

where $\rho(\omega)$ is the density of electron states, which is connected with the Green's function $G(\omega+\mathrm{i} 0)$ through the relation:

$$
\rho(\omega)=-\frac{1}{\pi} \operatorname{Im} G(\omega+\mathrm{i} 0) .
$$


Let us consider the diffusion contact of the crystal+impurity system with the reservoir. In that case, it fixes the chemical potential $\mu$ of the system.

Let us use a less rigid assumption than $W_{\alpha \beta}=$ const namely, $V_{\alpha \vec{k}}=$ const in $W_{\alpha \beta}$ (4). Then,

$$
W_{\alpha \alpha^{\prime}}(\omega)=\frac{\left|V_{\alpha \alpha^{\prime}}\right|^{2}}{2 \pi} \sum_{n} 2 \int_{0}^{\pi} \frac{\mathrm{d} k_{z}}{A-\alpha_{0} k_{z}^{2}}
$$

where

$$
\alpha_{0}=\hbar^{2} / 2 m_{z}^{*} d_{z}^{2}, \quad A=\omega-L_{0}-\hbar \omega_{c}\left(n+\frac{1}{2}\right), \quad\left|V_{\alpha \alpha^{\prime}}\right|^{2}=V^{*}(\alpha k) V\left(\alpha^{\prime} k\right) .
$$

According to $[9]$

$$
\int_{o}^{\pi} \frac{\mathrm{d} k_{z}}{A-\alpha_{0} k_{z}^{2}}=\left\{\begin{array}{lll}
\frac{1}{2 \sqrt{\alpha_{0} A}} \ln \left|\frac{\sqrt{\alpha_{0}} \pi+\sqrt{A}}{\sqrt{\alpha_{0}} \pi-\sqrt{A}}\right|, & A>0 \quad \text { (a) } \\
-\frac{1}{\sqrt{\alpha_{0} A}} \arctan \frac{\sqrt{\alpha_{0}} \pi}{\sqrt{A}}, & A<0 \quad \text { (b) }
\end{array}\right.
$$

Since $A$ depends on $\omega_{c}$ (and consequently, on the magnetic field), on $E_{0}$, and on the quantum number $n$, any changes thereof have an effect on the form and on the number of terms (11a) and (11b) in the summation sign (4). Such changes may be considered as:

$$
W_{\alpha \alpha^{\prime}}(\omega)=\frac{\left|V_{\alpha \vec{k}}\right|^{2}}{2 \pi \sqrt{\alpha_{0} A}}\left\{\sum_{n=0}^{N_{0}} \frac{1}{2} \ln \left|\frac{\sqrt{\alpha_{0}} \pi+\sqrt{A}}{\sqrt{\alpha_{0}} A-\sqrt{A}}\right|-\sum_{n=N_{0}+1}^{\infty} \arctan \frac{\sqrt{\alpha_{0}} \pi}{\sqrt{A}}\right\},
$$

where $N_{0}$ is the integer part of a number, which is a solution of the equation $A=0$, i.e.

$$
N_{0}=\left\|\frac{\omega-L_{0}}{\hbar \omega_{c}}-\frac{1}{2}\right\| .
$$

Here, denotation $\|x\|$ means the integer part of $x$.

It is obvious that the electron population of the impurity atoms is defined mainly by the electron states close to the electronic state $E_{0}$ of a free impurity. A similar assumption was used in the analysis of self-energy in the problem of the electron spectrum renormalization by the electron-phonon interaction in the normal metal $[10]$.

As it follows from the system of equations for $\left\langle n_{0}\right\rangle,\left\langle n_{1}\right\rangle$, there are possible not only solutions $\left\langle n_{0}\right\rangle=\left\langle n_{1}\right\rangle$ but at some conditions $\left\langle n_{0}\right\rangle \neq\left\langle n_{1}\right\rangle$ [8].

Consider the dependence of the uniform electron population of the impurity $\left\langle n_{0}\right\rangle,\left\langle n_{1}\right\rangle$ (i.e. $\left\langle n_{0}\right\rangle=\left\langle n_{1}\right\rangle=\langle n\rangle$ ) on the applied magnetic field at the range $10^{4}-10^{6}$ Oe. In figure 1 , the dependence $\langle n\rangle=f(H)$ is presented, where the electron state of the impurity $E_{0}$ is close to the chemical potential value $\left(E_{0}=0.149 \mathrm{eV}\right.$, $\mu=0.150 \mathrm{eV}$ ). The particular feature of such a dependence is the decline of $\langle n\rangle$ (on the whole) accompanied by sharp peaks at a certain value of the magnetic field. The appearance of the peaks is induced by the intersection of the chemical potential by 


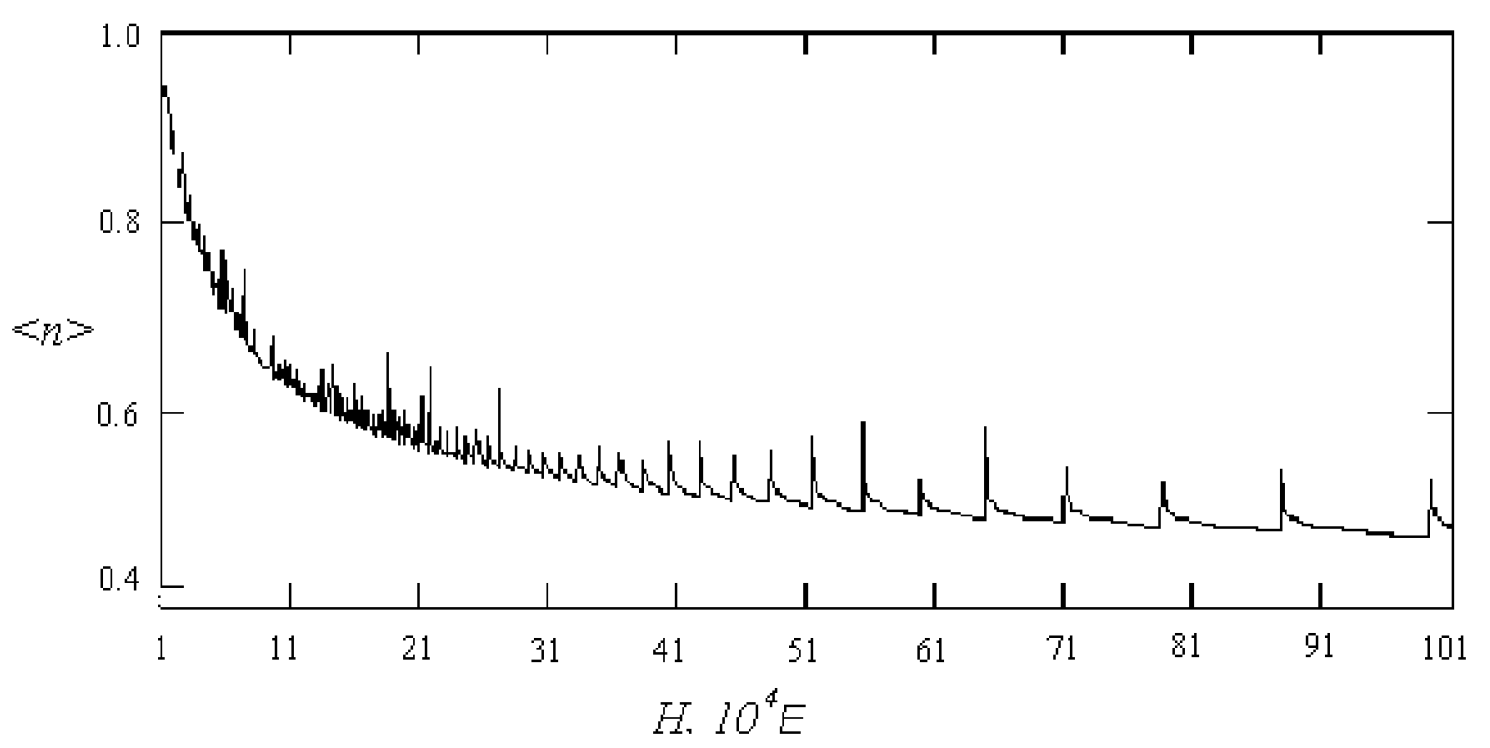

Figure 1. The value of the electron population of the impurity atoms at $T=$ $500 \mathrm{~K}\left(V_{00}=V_{11}=0.02 \mathrm{eV}, V_{10}=0.00, U=0.10 \mathrm{eV}, E_{0}=0.149 \mathrm{eV}\right)$.

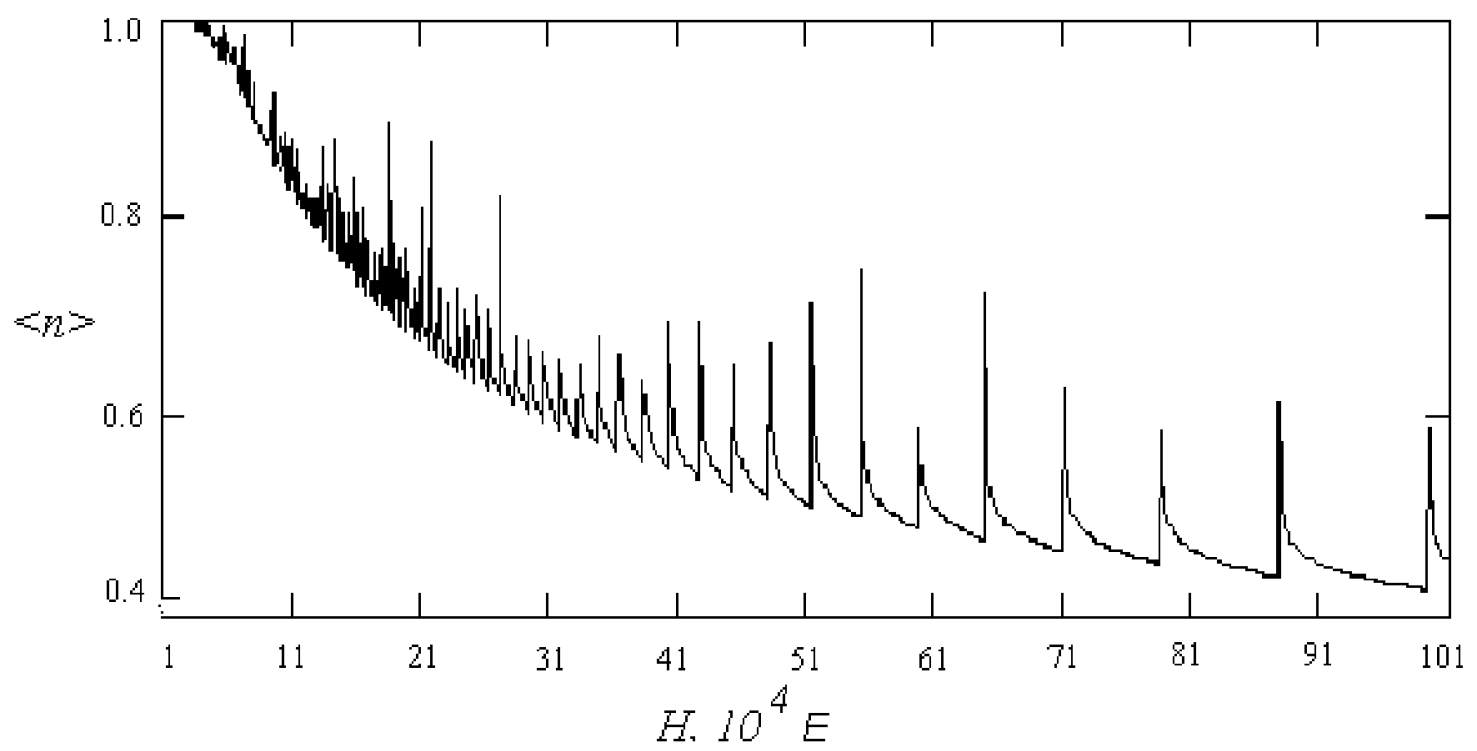

Figure 2. The value of the electron population of the impurity atoms at $T=$ $100 \mathrm{~K}$ and at the same parameters as in figure 1 except $E_{0}=0.10 \mathrm{eV}$. 
the Landau levels. The dependence of the levels on $H(\sim H)$ determines various values of the periods of jumps $\langle n\rangle$ at various $H$.

The dependence $\langle n\rangle=f(H)$ at the same parameters as in figure 1 but a bit lower position $E_{0}\left(E_{0}=0.10 \mathrm{eV}\right)$ qualitatively repeats the curve in figure 1 . Their quantitative differences are:

a) an obvious shift up in the curve in figure 2 to a greater value of electron population of the impurity atoms;

b) the change, namely, the increase of periods of $\langle n\rangle=f(H)$ jumps.

Alteration of $a_{0}$ and of the parameter of the Coulomb repulsion between the impurities $U_{0}$ has an inverse effect: the decrease of $\alpha_{0}$ or the increase of $U$ tends to decrease $\langle n\rangle$ only and to insufficiently change the form of $\langle n\rangle=f(H)$. In particular, the periods of jumps are the same in both cases.

The value of $\langle n\rangle=f(H)$ is more sensitive to temperature. Such a dependence at $T=100 \mathrm{~K}$ is presented in figure 2 . The form of the curve repeats the curve in figure 1 but it differs from the curve quantitatively: its value at low magnetic fields is greater and its growth is sharper but at high fields such a growth is smaller.

\section{The effect of the Green's function decoupling}

Microscopic studies of the intercalation and especially the adsorption have been extensively carried out using different theoretical approaches. Each of them has both advantages and disadvantages. The Heitler-London bonding without any charge transfer from impurity atoms into the crystal or vise-versa was used, in particular, in the induced-covalent-bond theory of the diatomic molecule adsorption [11]. However, this theory gives an incorrect result for the adsorption energy. In [12] it was shown that a more correct result can be obtained using the Hartree-Fock approximation. Still, even if this approximation correctly predicts the energy adsorption, the predicted charge transfer to the impurity atom is not compatible with experimental evidence from the change of workfunction and the equilibrium distance between the impurity and the crystal matrix.

There are discrepancies between different approaches in the Anderson's model. Consequently, it seems reasonable to analyze the effect of the Green's function decoupling.

Let us consider the Callen's decoupling widely used in the theory of magnetism (see, e.g., [13]):

$$
\left\langle\left\langle b_{f}^{+} b_{f} b_{f^{\prime}} \mid b_{q}^{+}\right\rangle\right\rangle=\left\langle n_{f}\right\rangle\left\langle\left\langle b_{f^{\prime}} \mid b_{q}^{+}\right\rangle\right\rangle+\chi\left\langle b_{f}^{+} b_{f^{\prime}}\right\rangle\left\langle\left\langle b_{f} \mid b_{q}^{+}\right\rangle\right\rangle,
$$

where $\chi$ is some arbitrary parameter, $b_{f}^{+}, b_{f}$ are Fermion operators ( $f$ is an index of radius-vector $\vec{R}_{f}$ ).

Such a decoupling may be considered, on the one hand, as the Tyablikov's decoupling, and, on the another hand, as the synthesized Hartree-Fock decoupling by introducing parameter $\chi$ therein [13]. 
It may be shown that the result of this decoupling can be presented as a formal change $W_{01} \rightarrow W_{01}+\chi U\left\langle a_{1}^{+} a_{0}\right\rangle$ and $W_{10} \rightarrow W_{10}+\chi U\left\langle a_{0}^{+} a_{1}\right\rangle$ in (3), which indicates the renormalization of the parameter, describing both crystal and impurity characteristics.

Correlators $\left\langle a_{0}^{+} a_{1}\right\rangle$ and $\left\langle a_{1}^{+} a_{0}\right\rangle$ can be obtained by a standard procedure, using corresponding Green's functions. Thus, we finally obtain the system of the transcendental equations with respect to correlators $\left\langle a_{0}^{+} a_{1}\right\rangle,\left\langle a_{1}^{+} a_{0}\right\rangle,\left\langle a_{0}^{+} a_{0}\right\rangle,\left\langle a_{1}^{+} a_{1}\right\rangle$.

Let us analyze the Callen's decoupling in the special case - infinitesimal parameter $\chi$ and $\left\langle a_{0}^{+} a_{0}\right\rangle=\left\langle a_{1}^{+} a_{1}\right\rangle=\langle n\rangle$. In this case

$$
\langle n\rangle=\frac{1}{2}\left\{f\left(\tilde{E}_{1}\right)+f\left(\tilde{E}_{2}\right)\right\},
$$

where $\tilde{E}_{1}, \tilde{E}_{2}, \tilde{K}$ are respectively $E_{1}, E_{2}, K$ after the mentioned change $W_{01} \rightarrow W_{01}+$ $\chi U\left\langle a_{1}^{+} a_{0}\right\rangle$ and $W_{10} \rightarrow W_{10}+\chi U\left\langle a_{0}^{+} a_{1}\right\rangle$.

After expanding the Fermi-Dirac distribution function in power series in $\chi$ up to the first order we obtain

$$
\langle n\rangle=\langle n\rangle_{\mathrm{HF}}+\frac{2 \chi U\left(W_{01}^{*}\left\langle a_{1}^{+} a_{0}\right\rangle+W_{10}\left\langle a_{0}^{+} a_{1}\right\rangle\right)}{\sqrt{\left(W_{00}-W_{11}\right)^{2}+4\left|W_{10}\right|^{2}}},
$$

where $\langle n\rangle_{\mathrm{HF}}$ is an average number population of the impurity in the Hartree-Fock approximation.

Thus, using a more common decoupling in comparison with the Hartree-Fock decoupling we come to a quantitative change of the average number of the population of the impurity only, namely, this number is greater.

\section{Discussion and conclusions}

The obtained results in the framework of various approximations indicate the possibility of a considerably nontrivial change of electron population of the impurity atoms by an applied quantizing magnetic field. As it follows from the self-consistent solution of the problem, such a change causes renormalization energy characteristics of the crystal+impurity system $-V_{\alpha \vec{k}}, U, E_{0}$ which determine, in particular, the kinetics of the intercalation or the adsorption, the number of the intercalated or adsorbed atoms. In other words, by change of the applied magnetic field - by its magnitude and the velocity of its change - one can actively effect the workfunction and the process on the whole. The final answer to these assumptions needs additional theoretical and experimental investigations.

The used model of the system with two impurity interacting atoms may be considered as a model of the crystal+molecule system. In this case we retain the electron structures of the atoms as a starting point for calculating the electron structure of the molecule. This is the so-called valence bond approximation. On the other hand, in the molecule orbital approach where the electrons do not belong to a single nucleus but to as many as may be energetically convenient. There are different opinions among the scientists about the usefulness of this or that approach. 
Herein above we presented some analogy between intercalation and adsorption, which permits to describe both effects by the same model. No doubt, this analogy has certain limitations. Consider some differences in those effects that produce nontrivial specific manifestations in concrete cases.

The problems connected with the crystal surface, especially in the applied studies, are more complicated than simple crystal model systems. Thus, the comprehensible standard techniques cannot be used in solving these problems. But within the framework of even a simple model and technique from the very start, as it was noted, it is necessary to solve a complicated eigenvalue problem. In other words, in the adsorption problem, the crystal surface should be taken into account by all means while in the intercalation problem, the crystal surface is unimportant. Such differences can be considered as the differences of the "dimensionality" of the systems.

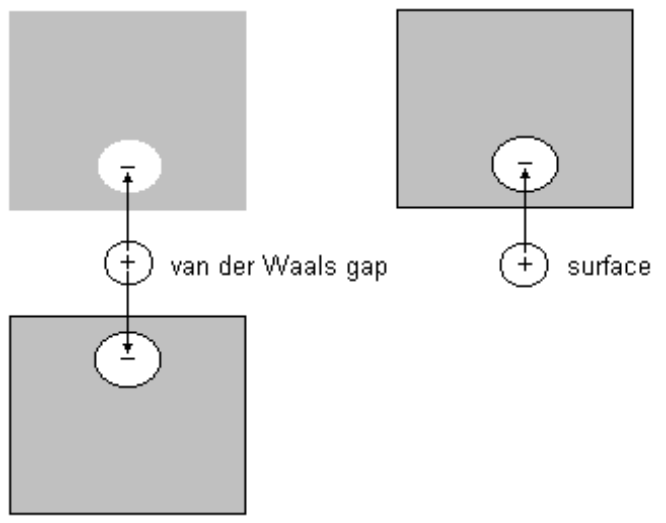

Figure 3. Schematic formation of a dipole in the case of (a) adsorption (the result is nonzero) and (b) intercalation (the resulting dipole equals zero).
Consider one of the effects caused by the dimensionality. In [1] the metalsemiconductor transition, induced by the adsorption of alkali metals on the surface in the $\mathrm{Na}, \mathrm{Cs} / \mathrm{Si}(001)$ systems, is described by the dipole interaction. The appearance of such dipoles is caused by the adsorption (see figure 3 ). However, in the intercalated layer crystal, a similar interaction is improbable since the resulting dipole moment caused by the charge transfer between the impurity and the crystal is equal to zero (see figure 3). Thus, in this case there is no dipole interaction.

\section{References}

1. Davydov S.Yu. // Fizika Tverdogo Tela, 2000, vol. 42, No. 6, p. 1129 (in Russian).

2. Yoffe A.D. // Solid State Ionics, 1983, No. 9-10, p. 59.

3. Grimley T.B. // Proc. Phys. Soc., 1967, vol. 90, No. 3. p. 751.

4. Bennett A.J., Falicov L.M. // Phys. Rev., 1966, vol. 151, No. 1, p. 512.

5. Wojcechowski K.F. // Acta Phys. Polon., 1966, vol. 29, No. 1, p. 119.

6. Newns D.M. // Phys. Rev., 1966, vol. 176, No. 5, p. 1123.

7. Anderson P.W. // Phys. Rev., 1961, vol. 124, No. 1, p. 41.

8. Lukiyanets B.A. // Phys. Stat. Sol. (b), 1991, vol. 163, No. 1, p. 171.

9. Prudnikov A.P., Brychkov Yu.A., Marychev O.I. Integrals and series. Moscow, Nauka, 1981 (in Russian).

10. Schrieffer J. Theory of Superconductivity. Moscow, Nauka, 1970 (in Russian).

11. Schrieffer J.R, Gomer R. // Surf. Sci., 1971, vol. 25, No. 1, p. 315.

12. Lyo S.K., Gomer R. // Phys. Rev. B, 1974, vol. 10, No. 11, p. 4161. 
13. Rudoj Yu.G. Modern state of the two-time Green-function method in quantum theory of magnetism. - In: Statistical Physics and Quantum Field Theory. ed. N.N. Bogolyubov. Moscow, Nauka, 1973, p. 97-185 (in Russian).

\section{Електронне заповнення домішкових атомів в кристалі в зовнішньому квантуючому магнітному полі}

\section{Б.А.Лукіянець}

Національний університет "Львівська політехніка", 79646 Львів, вул. Бандери, 12

Отримано 3 липня 2002 р.

Обговорюється модель, яка дозволяє описувати зарядовий стан домішкового атома як в явищі інтеркалювання шаруватого кристалу, так і в явищі адсорбції на кристалічній поверхні. В рамках моделі аналізується вплив зовнішнього квантуючого магнітного поля на величину електронного заповнення такого атома. Отримані результати дозволяють розглядати зовнішне магнітне поле як істотний фактор впливу на явище адсорбції і інтеркаляції.

Ключові слова: домішковий атом, інтеркаляція, адсорбція, квантуюче магнітне поле

PACS: $61.72 . B b, 68.35 . D$ 